\title{
Successful Treatment of Scalp Myxedema with Injected Hyaluronidase: A Case Report
}

\author{
Sébastien Menzinger ${ }^{a}$ Gürkan Kaya ${ }^{a}$ b \\ aDepartment of Dermatology, University Hospital of Geneva, Geneva, Switzerland; \\ ${ }^{b}$ Department of Clinical Pathology, University Hospital of Geneva, Geneva, Switzerland
}

\author{
Keywords \\ Myxedema - Graves' disease · Hyaluronidase
}

\section{Abstract}

Localized myxedema is an uncommon complication of Graves' disease. It is characterized by the accumulation of glycosaminoglycans, particularly hyaluronate. Here we describe the case of a 51-year-old female patient suffering from Graves' disease, who has presented for years a thickening of the scalp, consistent with myxedema, and successfully treated with repeated injections of hyaluronidase.

\section{Introduction}

Localized myxedema, also named thyroid dermopathy, is an uncommon complication of Graves' disease. The patients have high serum concentrations of thyroid-stimulating hormone (TSH) receptor antibodies and usually severe ophthalmopathy. It can also be associated with acropachy, of which the most common manifestation is clubbing. Both dermopathy and ophthalmopathy are characterized by the accumulation of glycosaminoglycans, particularly hyaluronate (HA), a major constituent of the extracellular matrix of the skin. Histologically, myxedema shows large amounts of mucin in the dermis with fragmentation of collagen fibers. The pathophysiology of localized myxedema is partially understood. An excessive production 

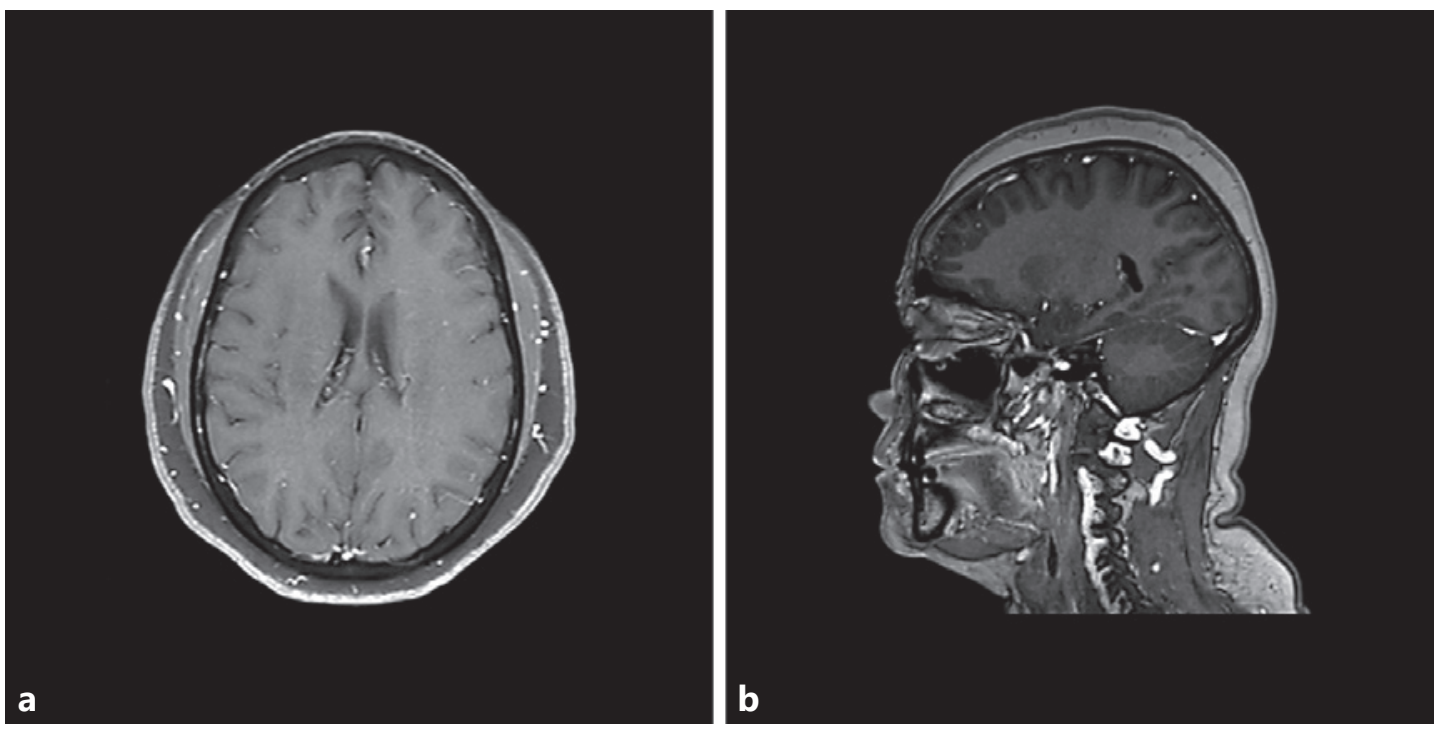

Fig. 1. Cerebral-cranial MRI. Transverse plane (a) and sagittal plane (b). Thick and boggy scalp.

of HA resulting from the activation of fibroblasts may be the main pathological process. It is likely that the interaction of TSH receptor antibodies and TSH receptor on fibroblasts plays an important role. The lesions of localized myxedema occur usually in the pretibial area, but cases of involvement of the upper extremities, upper back, shoulders and nose are reported [1]. To our knowledge, localized myxedema of the scalp, proven by histology, in the context of Graves' disease, has never been reported. However, it has been reported twice in hypothyroid patients: in 1964 by Frankel et al. [2] and in 1999 by Corbalan-Velez et al. [3], as a cutis verticis gyrata secondary to a hypothyroid myxedema.

In our opinion, scalp myxedema must be differentiated from cutis verticis gyrata and lipedematous alopecia/lipedematous scalp. Cutis verticis gyrata is a skin condition characterized by skin hypertrophy with convoluted folds and deep furrows that resemble the outer surface of the cerebrum $[4,5]$. It is characterized histologically by a diffuse thickening of the dermis, and sometimes the subcutaneous fat, with thick collagen bundles and sometimes with hyperplasia of adnexal structures. Lipedematous scalp and lipedematous alopecia are rare diseases of unknown etiology showing a thick, boggy scalp with varying degrees of hair loss and no clearly associated medical disorder. It is reported essentially in female patients [6]. Histologically, thickening of the subcutaneous fat with massive localized edema is observed. There is usually no accumulation of mucin $[7,8]$.

The first step in the management of myxedema usually consists of normalizing thyroid function. In case of symptoms or cosmetic concerns, topical corticosteroids are usually used. Systemic options, rarely needed, comprise systemic corticosteroids, plasmapheresis, intravenous immunoglobulins and octreotide [1]. We also reported the case of a patient with pretibial myxedema, successfully treated with intralesional injections of hyaluronidase [9].

Hyaluronidases are endo- $\beta$ - $\mathrm{N}$-acetyl-hexosaminidases essential for the breakdown of HA [9] and are essentially used to adjust misplacement of HA fillers. Many case reports have shown the efficacy of hyaluronidases in reducing unwanted deposits of HA, with very few adverse reactions [10-12].

Here we describe the case of a patient with Graves' disease, who had complained of scalp thickening for years, which was consistent with myxedema and treated successfully with repeated injections of hyaluronidase. 

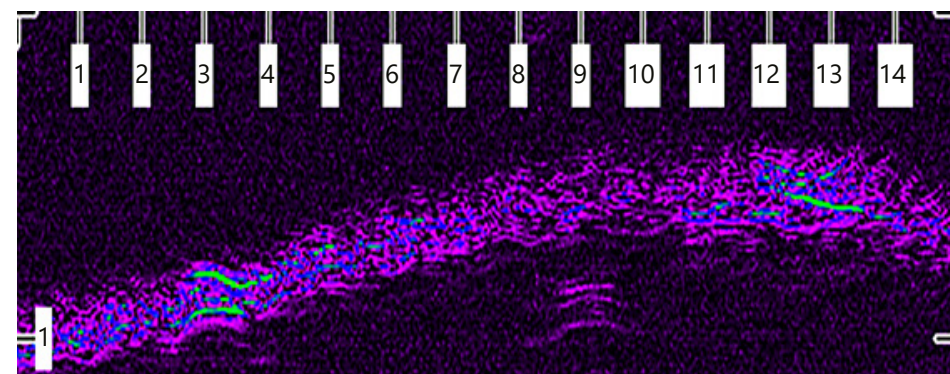

Fig. 2. Dermal-epidermal thickness measured by high-frequency ultrasonography, on day 0 .

\section{Case Report}

A 51-year-old woman was referred to our clinic, complaining of scalp thickening for years, associated with paroxystic intermittent episodes of painful swelling. She was known for Graves' disease treated with carbimazole. On physical examination, the whole scalp was thickened, soft, nontender and without alopecia or any other skin changes. Blood tests 

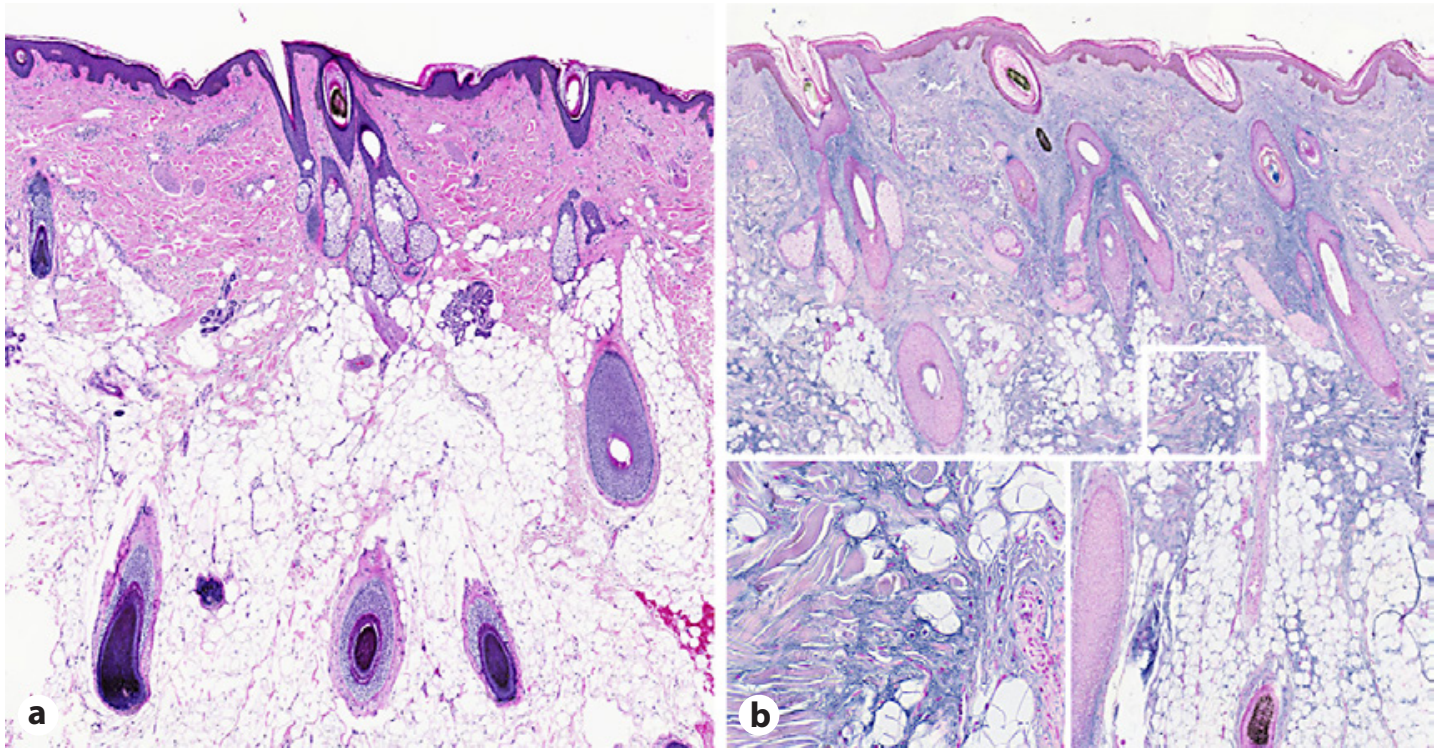

Fig. 3. Histology. a HE $\times 20$. Collagen bundles in the superficial, mid and deep dermis are sometimes surrounded by clear spaces. $\mathbf{b}$ Colloidal iron. Magnification $\times 20$ (inset $\times 100$ ). Deposition of interstitial mucinous material in the dermis and the superficial part of the subcutis.

Fig. 4. Evolution of scalp thickness during the follow-up. Arrows correspond to hyaluronidase injections.

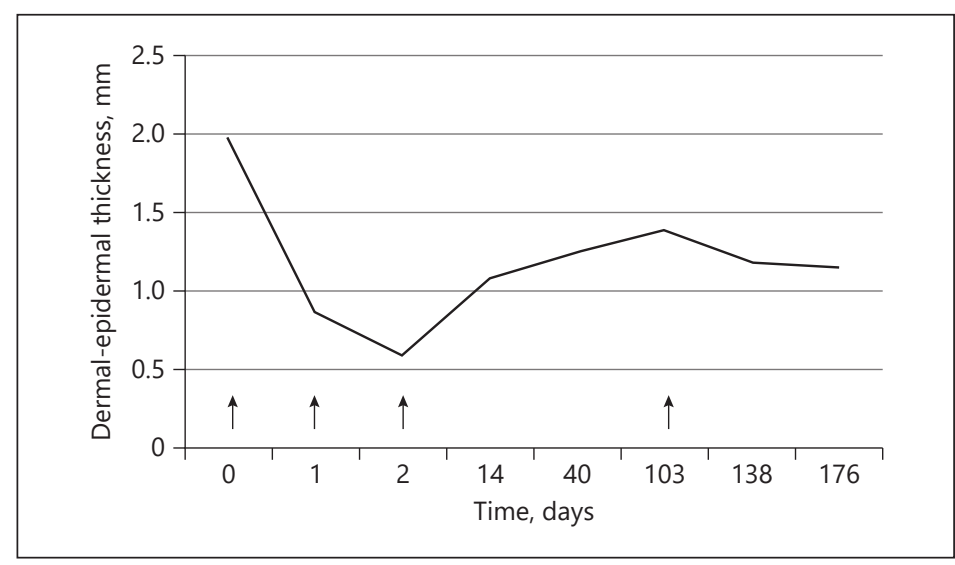

revealed a normal TSH, but elevated titers of anti-TSH receptor, antithyroperoxidase and antithyroglobulin autoantibodies. Cerebral-cranial MRI showed a diffuse thickening of the cutaneous and subcutaneous tissue of the scalp (Fig. 1). High-frequency ultrasound showed an abnormal thickness of the skin, with an initial measure of $1.97 \mathrm{~mm}$ in the right occipitoparietal area (Fig. 2). We performed a large and deep biopsy, and the histological analysis was consistent with myxedema (Fig. 3).

We decided to treat the patient with injected hyaluronidase. On day 0 , we performed intradermal injections of 30 IU of hyaluronidase (Hylase Dessau, Germany) in 4 different points (= $120 \mathrm{IU}$ ) in the right occipitoparietal area without any complication. As mentioned above, the initial thickness was $1.97 \mathrm{~mm}$. On day 1, we observed a dramatic improvement with a decrease in skin thickness to $0.86 \mathrm{~mm}$ ultrasonographically, and clinically the skin seemed more depressible. We decided to repeat the injections (120 IU). On day 2, we observed a decrease to $0.59 \mathrm{~mm}$, with a greater impression of depressibility. On day 14 the patient was 
very satisfied with the treatment, had no immediate or late complications, and did not feel any paroxystic painful swelling during this interval. The thickness was $1.08 \mathrm{~mm}$. We noted also a better depressibility of the skin on the rest of the scalp. We repeated the injections at the same site (120 IU). On day 40, the thickness was 1.25 , and $1.39 \mathrm{~mm}$ on day 103 . The patient felt again few paroxystic intermittent episodes of painful swelling. We continued the injections (120 IU). We observed a decrease in the thickness to 1.18 and $1.15 \mathrm{~mm}$ on days 138 and 176, respectively, with a decrease in painful sensation. The evolution of scalp thickness measured ultrasonographically is described in Figure 4.

\section{Discussion}

We have already reported that pretibial myxedema can be improved by the intralesional injections of hyaluronidase [9]. Here we report the case of a patient with scalp myxedema, a very uncommon manifestation of Graves' disease, which also improved by repeated injections of hyaluronidase, without any adverse effects, and with a follow-up of 176 days. It was also interesting to observe a diffuse effect on the whole scalp, although the injections were always performed at the same site (right occipitoparietal area). Based on this observation, we can hypothesize that hyaluronidase spreads into the whole scalp after injections.

Effectiveness of injected hyaluronidase for the treatment of myxedema has already been reported by others. Grais [13] was the first to describe the successful use of hyaluronidase for the treatment of pretibial myxedema in 1949, before Rosman [14] in 1950 and Paver [15], who published a case series with 4 patients with pretibial myxedema in 1977, treated with topical corticosteroids only on the upper half of the leg and with injections of hyaluronidase and topical corticosteroids on the lower half. One week later, only the area treated with hyaluronidase injections was cleared [15]. Recently, Hoesly et al. [16] have also reported its efficacy in 2 patients. All these case reports suggest a very good efficacy of injected hyaluronidase for the treatment of localized myxedema. We did not observe any side effects during the treatment, and usually very few adverse reactions (erythema, pruritus) are described [10$12]$. We need larger trials to determine the actual effectiveness of this treatment in this indication and its safety profile.

\section{Acknowledgments}

We thank Prof. J.-H. Saurat for addressing the patient for hyaluronidase treatments and his critical reading of the manuscript.

\section{Statement of Ethics}

This study complies with the guidelines for human studies and animal welfare regulations. The authors state that the subject has given her informed consent. Hylase Dessau is approved for subcutaneous injections.

\section{Disclosure Statement}

The authors have no conflicts of interest to declare. 
Menzinger and Kaya: Hyaluronidase Treatment for Scalp Myxedema

\section{Author Contributions}

S.M. contributed to the acquisition, analysis and interpretation of data for the work, participated in drafting the work, approved the final version to be published and agreed to be accountable for all aspects of the work in ensuring that questions related to the accuracy or integrity of any part of the work are appropriately investigated and resolved

G.K. made substantial contributions to the conception and design of the work and to the acquisition, analysis and interpretation of data for the work, participated in drafting the work, revising it critically for important intellectual content, approved the final version to be published and agreed to be accountable for all aspects of the work in ensuring that questions related to the accuracy or integrity of any part of the work are appropriately investigated and resolved.

\section{References}

1 Fatourechi V. Pretibial myxedema: pathophysiology and treatment options. Am J Clin Dermatol. 2005;6(5): 295-309.

2 Frankel EB, Frankel AR. Localized myxedema of the scalp and hypothyroidism. Arch Dermatol. 1964 Nov; 90(5):460-2.

3 Corbalán-Vélez R, Pérez-Ferriols A, Aliaga-Bouiche A. Cutis verticis gyrata secondary to hypothyroid myxedema. Int J Dermatol. 1999 0ct;38(10):781-3.

4 Dumas P, Medard de Chardon V, Balaguer T, Cardot-Leccia N, Lacour JP, Lebreton E. [Primary essential cutis verticis gyrata: case report and literature review]. Ann Chir Plast Esthet. 2010 Jun;55(3):243-8. French.

5 Lamelas AM, Taub PJ, Silver L, Kressel A. Congenital primary essential cutis verticis gyrata. Eplasty. 2016 Apr; 16:ic13.

6 Yaşar S, Mansur AT, Göktay F, Sungurlu F, Vardar Aker F, Ozkara S. Lipedematous scalp and lipedematous alopecia: report of three cases in white adults. J Dermatol. 2007 Feb;34(2):124-30.

7 Fair KP, Knoell KA, Patterson JW, Rudd RJ, Greer KE. Lipedematous alopecia: a clinicopathologic, histologic and ultrastructural study. J Cutan Pathol. 2000 Jan;27(1):49-53.

8 Martín JM, Monteagudo C, Montesinos E, Guijarro J, Llombart B, Jordá E. Lipedematous scalp and lipedematous alopecia: a clinical and histologic analysis of 3 cases. J Am Acad Dermatol. 2005 Jan;52(1):152-6.

9 Menzinger S, Kaya A, Saurat JH, Kaya G. Injected hyaluronidase reduces the volume of exogenous hyaluronate fillers in mice and results in clinical improvement in a patient with pretibial myxedema. Dermatopathology (Basel). 2016 Jul;3(3):61-7.

10 Hirsch RJ, Narurkar V, Carruthers J. Management of injected hyaluronic acid induced Tyndall effects. Lasers Surg Med. 2006 Mar;38(3):202-4.

11 Soparkar CN, Patrinely JR, Tschen J. Erasing restylane. Ophthal Plast Reconstr Surg. 2004 Jul;20(4):317-8.

12 Pierre A, Levy PM. Hyaluronidase offers an efficacious treatment for inaesthetic hyaluronic acid overcorrection. J Cosmet Dermatol. 2007 Sep;6(3):159-62.

13 Grais ML. Local injections of a preparation of hyaluronidase in the treatment of localized (pretibial) myxedema. J Invest Dermatol. 1949 Jun;12(6):345-8.

14 Rosman D. The treatment of localized (pretibial) myxedema with hyaluronidase. NY State J Med. 1950 Aug; 50(16):1939-42.

15 Paver WK. The treatment of pretibial myxoedema and cutaneous myxoid cysts. Australas J Dermatol. 1977 Dec;18(3):144-5.

16 Hoesly PM, Tolaymat LM, Sluzevich JC, Keeling JH. Pretibial myxedema successfully treated with intralesional hyaluronidase. JAAD Case Rep. 2018 Oct;4(9):874-6. 